\title{
Satellite Dynamics Simulator Development Using Lie Group Variational Integrator
}

\author{
Daeyoung Lee * John C. Springmann, and Sara C. Spangelo* and James W. Cutler ${ }^{\dagger}$ \\ Aerospace Engineering, University of Michigan, Ann Arbor, Michigan, 48109, USA
}

\begin{abstract}
Simulation technology is becoming increasingly crucial in the design and optimization of satellites due to the difficulties in testing and verifying system parameters on the ground. Computationally tractable and accurate methods are required in order to test satellite parameters in the complex and dynamic space environment. Although various satellite teams have developed simulation tools, many suffer from inaccurate numerical integrators, resulting in their simulations being of low fidelity for long duration simulations. This paper presents a MATLAB/Simulink-based simulator which includes high fidelity integration and modeling for accurate and relatively quick results. The simulator includes an energy-preserving variational integrator for both translational and rotational dynamics. A Lie Group Variational Integrator is used for the rotational dynamics, which enforces an orthogonality constraint for improved accuracy. This approach requires less computational time relative to other integration methods such as Runge-Kutta method for the same level of integration accuracy. The simulator includes perturbations to the orbital motion and attitude, including Earth oblateness, aerodynamic drag, solar pressure, gravity gradient, and residual dipole. The simulator also includes an advanced hysteresis model for improved modeling of magnetic attitude control systems. Simulation results are provided for a representative small satellite mission in low earth orbit with a passive magnetic stabilization control system. We compare the novel integration and hysteresis techniques to conventional simulators for long duration simulations for a realistic mission scenario.
\end{abstract}

\section{Introduction}

Spacecraft system design relies on modeling and simulation tools. Simulation is a critical component of the system design to verify the vehicle and operational design parameters that are difficult to verify with ground-based testing. The development of a simulation tool which achieves more accurate results will aid the design of more reliable and capable satellites. However, high-fidelity simulations are hard to achieve because of the accumulation of errors in the numerical integration of the rigid body dynamics. In this paper, we have developed a MATLAB/Simulink-based simulator which utilizes variational integration to achieve high-accuracy results for long-duration simulations.

Currently, there are various simulation tools available for satellite design. For example, Satellite Tool Kit (STK), developed by Analytical Graphics Incorporated, is an excellent tool that can be used for various parts of the satellite design process. However, one drawback of STK and many other commercial simulators developed in MAT$\mathrm{LAB} /$ Simulink or $\mathrm{C}++$ is that they only provide users with the restricted customizeability. Other configurable simulators, such as CubeSim, are prone to numerical integration errors, which result in decreased simulation accuracy as the duration of the simulation increases. A small sample of existing simulators include References 1-5.

The simulator developed in this paper utilizes the Lie Group Variational Integrator (LGVI), ${ }^{6}$ the high-fidelity magnetic hysteresis modeling, ${ }^{7}$ and also includes disturbance forces and moments. The LGVI method preserves the orthonormal constraint on the attitude matrix and also preserves energy. This results in a higher accuracy simulation when compared to typical methods such as quaternion propagation via Runge-Kutta (RK) integration and brute-force quaternion normalization. The LGVI method is also advantageous when considering computation time because the calculation time is faster than RK methods for similar total energy error. For example, to get a similar energy conservation level, if the integration calculation was performed with 0.001 second of time intervals over 30 seconds, it is verified that the LGVI requires over 10 times less CPU time than the $\mathrm{RK}^{6}$ in the rotational motion simulation. The combination of these advantages make this an improved simulator for long duration testing. A natural use of the simulator is for the design and testing of passive magnetic attitude control systems because such systems can take anywhere

\footnotetext{
*Graduate Student, Aerospace Engineering, University of Michigan, Ann Arbor, MI 48109, AIAA Student Member.

${ }^{\dagger}$ Assistant Professor, Aerospace Engineering, University of Michigan, Ann Arbor, MI 48109, AIAA Member.
} 
from days to months to reach the steady state behavior. To further facilitate use with passive magnetic control systems, we utilize a high fidelity hysteresis model. ${ }^{7}$

This simulator has been used in the design of the Radio Aurora Explorer (RAX) nanosatellite. ${ }^{8}$ Although RAX uses a passive magnetic attitude control system, the control portion of the simulator is modular so a user can implement a custom control algorithm. The combination of reduced integration errors, a higher fidelity hysteresis model, and the ability to implement custom control laws makes this an ideal simulator for use by various satellite developers.

In this paper, we present the simulator structure, the implementation of translational and rotational dynamics with the LGVI integration, the modeling and results of the space environment and disturbances, and also provide comparisons to RK integration.

\section{Simulator Structure}

The structure of our simulator is shown in Fig. 1. In this figure, arrowed lines indicate the information transferred between subroutines. This simulation is based on a discrete time; at every time interval, each block updates and the results are propagated through the simulator. First, in the orbit dynamics part (left center), the satellite position is generated using the orbit propagator. With this position information, it is also possible to calculate the magnetic vector of the Earth and Sun position vectors, the magnetic moment, the hysteresis strip moment, and various disturbance moments and orbital perturbation forces. These moments and orbital perturbation forces are fed back to the orbit and attitude dynamics block of the simulator and used to calculate future dynamics. In our simulator, a solar cell power generation model and a communication model are included. Given the satellite's position and attitude, the simulator estimates the power generated by the solar panels and the ground station link opportunities. The novel element of the developed simulator is the LGVI algorithm which is used to calculate the motion of the satellite. The simulator blocks are described in the following sections.

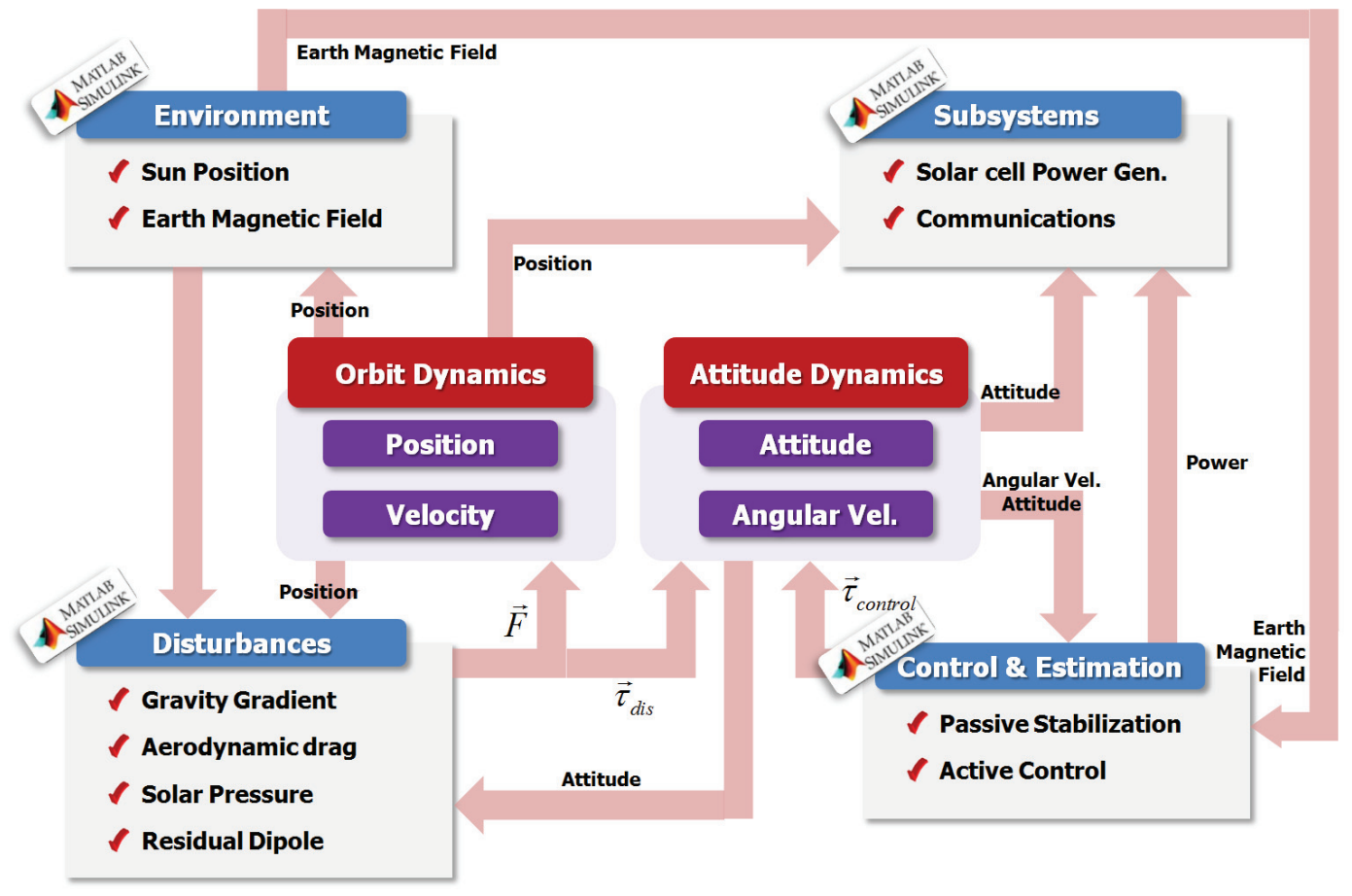

Figure 1. The structure of the simulator.

\section{Orbit Dynamic and Attitude Dynamics}

The orbital dynamics of the simulator are based on Newton's law and the spacecraft attitude is based on Euler's equation for rigid body rotation.

In section III.A, we introduce necessary frames. After that, the orbit dynamics is described in section III.B and 
the satellite attitude dynamics and kinematics based on rigid body dynamics will be introduced in section III.C, and finally, in section III.D, we decide the simulator integrator as LGVI.

\section{A. Frames}

It is convenient to represent the attitude information of the satellite in different reference frames. To provide clarity, we define and describe our use of reference fames. In the following list of the frames, frame notation for the mathematical expressions are denoted in the parenthesis. Subscripts are used. For example, $\left.\vec{r}\right|_{A}$ indicates the vector $\vec{r}$ is resolved in frame $A$. Frame notations are always expressed with capital letters.

- Inertial Frame (denoted as I) : The inertial frame is a non-accelerating frame in which Newton's laws of motion apply. We use the conventional Earth-centered inertial frame, for which the origin is located at the center of Earth.

- The Earth-centered Earth-fixed frame (ECEF, denoted as E) : The ECEF frame also has its origin at the center of the Earth, but this frame rotates with the Earth. As a result, the coordinates of a point fixed to the surface of the Earth do not change.

- Local Vertical and Local Horizontal frame (LVLH, denoted as L) : The LVLH frame has its origin at the satellite's center of mass. The $\mathrm{x}$-axis points in the velocity direction, the z-axis points to the Earth center, and the $\mathrm{y}$-axis completes the right-hand orthogonal rule.

- Body frame (denoted as B) : The body-fixed reference frame is a moving coordinate frame fixed to the satellite. The axes are fixed in the satellite where each axes completes the right-hand orthogonal system. The origin is located at the center of mass.

- The orientation matrix between frames: The orientation matrix $O$ from frame $B$ relative to frame $A$ is denoted $O_{B / A}$. This matrix is an element in $\mathrm{SO}(3)$, which is defined by Eq. (1)

$$
S O(3)=\left\{O \mid O \in \mathbb{R}^{3 \times 3}, O^{T} O=I \quad \text { and } \quad \operatorname{det}(O)=1\right\}
$$

where $\mathbb{R}^{3 \times 3}$ is the set of all $3 \times 3$ matrices with real elements and $I$ is the $3 \times 3$ identity matrix. In general, the frame change of a vector from one frame to another, can be written,

$$
\left.\vec{v}\right|_{B}=\left.O_{B / A} \cdot \vec{v}\right|_{A}
$$

The orientation matrix satisfies Eq. (3)

$$
O_{B / A}=\left(O_{B / A}\right)^{-1}=\left(O_{A / B}\right)^{T}
$$

$O_{B / I}$ is the orientation matrix from the inertial(I) to the body-fixed frame(B), $O_{E / I}$ is from the inertial frame(I) to the ECEF frame(E) and $O_{B / E}$ is from the ECEF frame(E) to the body-fixed frame(B). Because LGVI calculation result is expressed with $O_{B / I}$, this orientation matrix becomes a base for the calculation of other relationships between frames. Fig. 2 presents these relationships between frames.

\section{B. Satellite Orbit Dynamics}

Variational integrators are momentum-preserving and symplectic. ${ }^{9}$ However, variational integrators can only be defined for Hamiltonian systems derived from the Euler-Lagrange equations of a discretized Hamilton's principle. Because of this, Lee et al. ${ }^{9}$ derived satellite orbital dynamics with a form of the discrete equations in Hamiltonian form. ${ }^{9}$ The discrete equations of motion for satellite, in Hamiltonian form, are given by Eq. (4). ${ }^{9}$

$$
\vec{x}_{k+1}=\vec{x}_{k}+\frac{h}{m} \vec{\gamma}_{k}-\frac{h^{2}}{2 m} \frac{\partial U_{k}}{\partial \vec{x}_{k}}, \quad \vec{\gamma}_{k+1}=\vec{\gamma}_{k}-\frac{h}{2} \frac{\partial U_{k}}{\partial \vec{x}_{k}}-\frac{h}{2} \frac{\partial U_{k+1}}{\partial \vec{x}_{k+1}}
$$

Here, $h$ is a time interval, $m$ is a mass of satellite and $\frac{\partial U_{k}}{\partial \vec{x}_{k}}$ is a variation of potential Energy. The variable $\vec{x}_{k}$ and $\vec{\gamma}_{k}$ can be transfered to the position and velocity of satellite $\vec{r}_{I}$ and $\vec{v}_{I}$ with Eq. (5)

$$
\vec{r}_{I}=\vec{x}_{k}, \quad \vec{v}_{I}=\frac{1}{m} \cdot \vec{\gamma}_{k}
$$




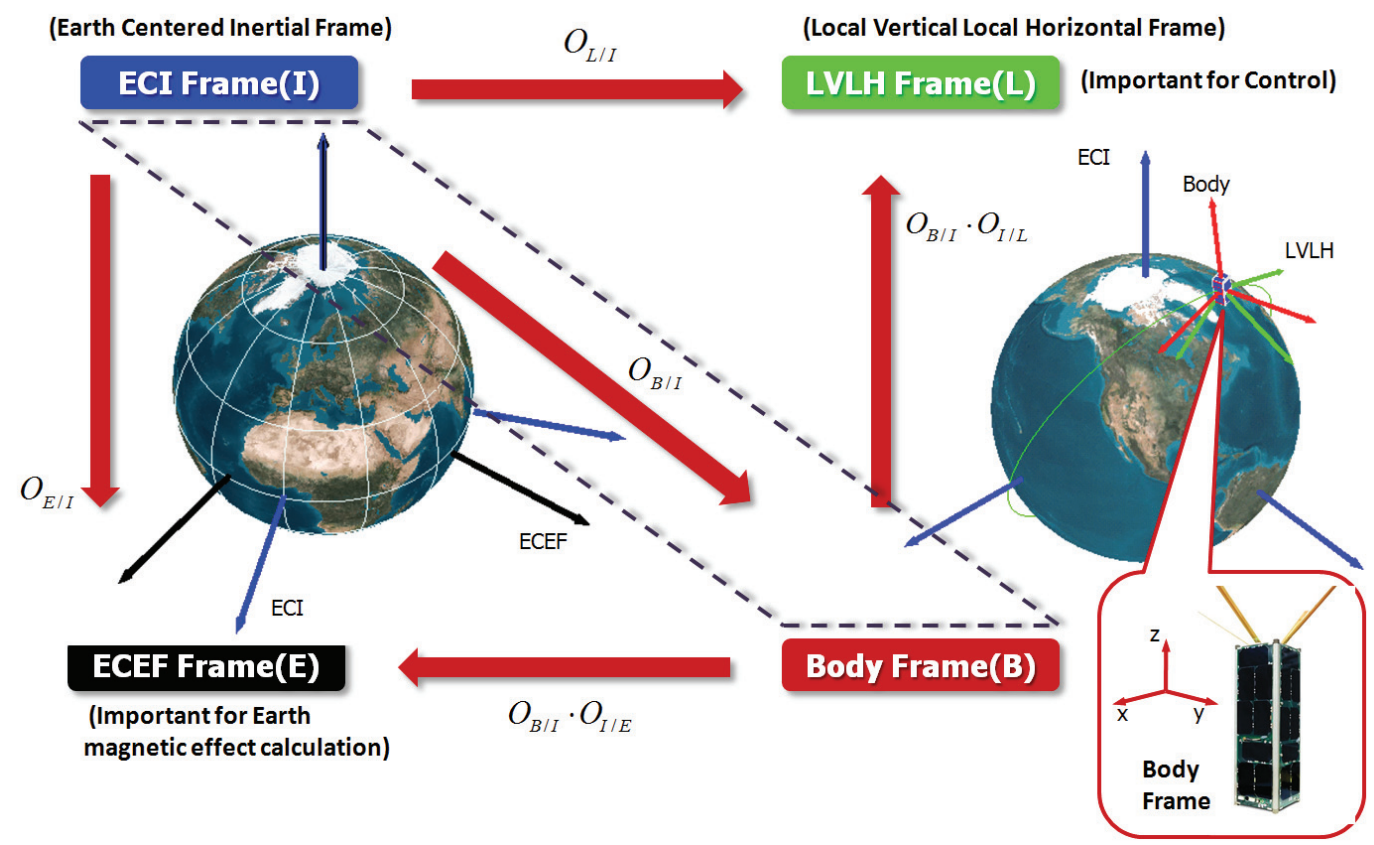

Figure 2. The relation between the frames.

\section{Satellite Rotational Dynamics and Kinematics}

\section{Dynamics and Kinematic Equation}

We assume the satellite is an ideal rigid body as this simplifies the modeling. For future development, more realistic model can be adopted. The dynamic model of the satellite is derived using the Newton-Euler formulation, where the angular momentum change is related to applied moments. The satellite rotational dynamic model is:

$$
\left.J \dot{\vec{\omega}}_{B / I}\right|_{B}+\left.\vec{\omega}_{B / I}\right|_{B} \times\left(\left.J \vec{\omega}_{B / I}\right|_{B}\right)=\left.\vec{M}\right|_{B}
$$

where $J$ is the $3 \times 3$ inertia matrix, $\left.\vec{\omega}_{B / I}\right|_{B}$ is the angular velocity of the satellite relative to the inertial frame expressed in the body frame, and $\left.\vec{M}\right|_{B}$ is the torque acting on the satellite, also resolved in body frame. The kinematics describe the satellite's orientation in space and are given by Eq. (7),

$$
\dot{O}_{B / I}+S\left(\left.\vec{\omega}_{B / I}\right|_{B}\right) O_{B / I}=0
$$

where $O_{B / I}$ is the orientation matrix describing the body relative to the inertial frame, $\left.\vec{\omega}_{B / I}\right|_{B}$ is the angular velocity of the satellite relative to the inertial frame expressed in the body frame and $S(\cdot)$ means the skew symmetric matrix. ${ }^{9}$

\section{Lie Group Variational Integrator (LGVI)}

Numerical integration algorithms have been developed for Hamiltonian systems defined on configurations with a Lie group structure by Lee et al. ${ }^{6}$ This approach is guaranteed to preserve a energy property and to maintain the Lie group structure, even over long time periods. A specific Lie group variational integrator for the satellite attitude models has been developed with MATLAB/Simulink. This Lie group variational integrator provides confidence that the computed simulation results for the satellite attitude dynamics are accurate, even over many orbits. ${ }^{6}$ This LGVI is based on the Lie group integrator developed by Iserless et al. ${ }^{10}$ and this Lie group numerical integration method is given by the following integration rule,

$$
R_{k+1}=\exp \left(-h S\left(\frac{\vec{\omega}_{k}+\vec{\omega}_{k+1}}{2}\right)\right) R_{k}
$$

where $R_{k} \in S O(3)$, and $\vec{\omega}_{k} \in \mathbb{R}^{3}$ is the angular velocity of the rigid body in the body fixed frame and $h \in \mathbb{R}$ is the constant integration step size, and the subscript $k$ denotes discrete time step at $t=k h$. To calculate the angular 
velocity, we can integrate the angular velocity and it is possible by choosing any of two different numerical methods: a Runge-Kutta method or a variational integrator. However, only the variational integrator preserves the energy property well, and Lee et al. ${ }^{6}$ mixed this variational integrator concept to Lie group integrator which is useful for the kinematic equation. The variational integrator is given by the following integration rule,

$$
\begin{gathered}
S\left(h J \vec{\omega}_{k+1}\right)=F_{k} J_{d}-J_{d} F_{k}^{T}, \\
J \vec{\omega}_{k+1}=F_{k}^{T} \vec{\omega}_{k} .
\end{gathered}
$$

where $F_{k} \in S O(3)$, the relative attitude between two integration steps, is obtained by solving the linear algebraic matrix equation, and $J_{d} \in \mathbb{R}^{3 \times 3}$ is a nonstandard moment of inertial matrix defined by $J_{d}=\frac{1}{2} \operatorname{tr}\left[J_{d}\right] I_{3 \times 3}-J .{ }^{11}$ The variational integrator preserves both of the conserved quantities to essentially computer supporting precision of the computer floating point operation.

\section{Simulation of the Orbit Environment}

In this section, the environmental models used in the simulator including Earth's magnetic field, sun position, and eclipse models are presented.

\section{A. Earth Magnetic Field}

The 2005 International Geomagnetic Reference Field (IGRF) ${ }^{12}$ is used to determine the local Earth magnetic field vector at all points within the satellite orbit. This model is given by Eq. (11),

$$
\vec{V}=a \sum_{n=1}^{N} \sum_{m=0}^{n}\left(\frac{a}{r}\right)^{n+1}\left(g_{n}^{m} \cos m \phi+h_{n}^{m} \cos m \phi\right) P_{n}^{m}(\cos \theta)
$$

where $a$ is the mean radius of the Earth $(6371.2 \mathrm{~km})$, and $r, \phi, \theta$ are the geocentric spherical coordinates. $r$ is the distance from the center of the Earth, $\phi$ is the longitude eastward from Greenwich, and $\theta$ is the colatitudes equal $90^{\circ}$ minus the latitude. $P_{n}^{m}(\cos \theta)$ are Schmidt quasi-normalized associated Legendre functions of degree $n$ and order $m$, where $n \geq 1$ and $m \leq n$. The maximum spherical harmonic degree of the expansion is $N$. Together with the orbit propagator, an estimate of the magnetic field can be made from IGRF model in ECEF frame.

\section{B. Sun Position and Eclipse}

Sun position and eclipse model is important for solar pressure disturbance moment calculations.

\section{Solar Model}

If the direction of the sun is known, it provides a well-defined reference vector that can be utilized in the satellite's attitude estimation. This vector is also required to estimate solar pressure. To be able to estimate the sun's direction vector, the relationship between the Earth and the sun has to be known. It is obvious that the Earth revolves around the sun, but it is more convenient to describe the relationship from the Earth's point of view, as illustrated in the Fig. 3, because inertial frames for the satellite motion is Earth-centered. The elevation, $\varepsilon_{S}$, of the sun from the Earth's equator varies by $\pm 23^{\circ}$. Kristiansen et al. ${ }^{13}$ proposed the following relationship to calculate the solar elevation:

$$
\varepsilon_{s}=\frac{23 \pi}{180} \sin \left(\frac{2 \pi}{365} T_{s}\right)
$$

where $T_{s}$ is the time elapsed since the first day of spring. The sun's position, $\lambda_{S}$ (See Fig. 4), in this imaginary orbit around the sun, is given by :

$$
\lambda_{s}=\frac{2 \pi}{365} T_{s}
$$

Knowing the elevation, $\varepsilon_{S}$, and Sun's position in relation to the Earth, it is possible to calculate a vector pointing to the sun. The calculation, starts with the initial position given on the first day of spring (vernal equinox).

$$
\left.\hat{S}_{0}\right|_{I}=\left[\begin{array}{lll}
1 & 0 & 0
\end{array}\right]^{T}
$$




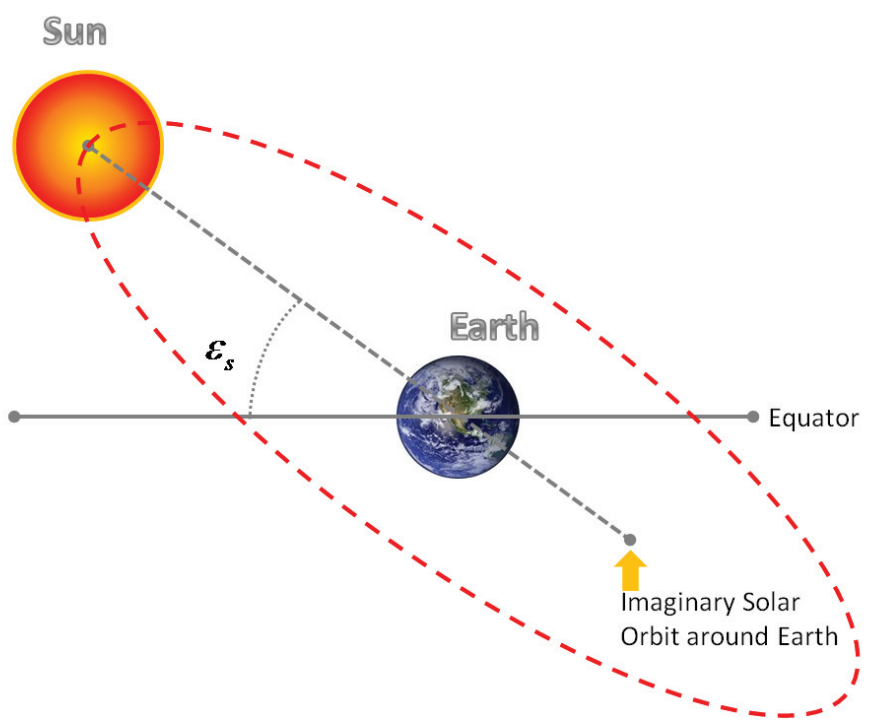

Figure 3. Sun elevation in an imaginary orbit around the earth, adapted from Reference 5.

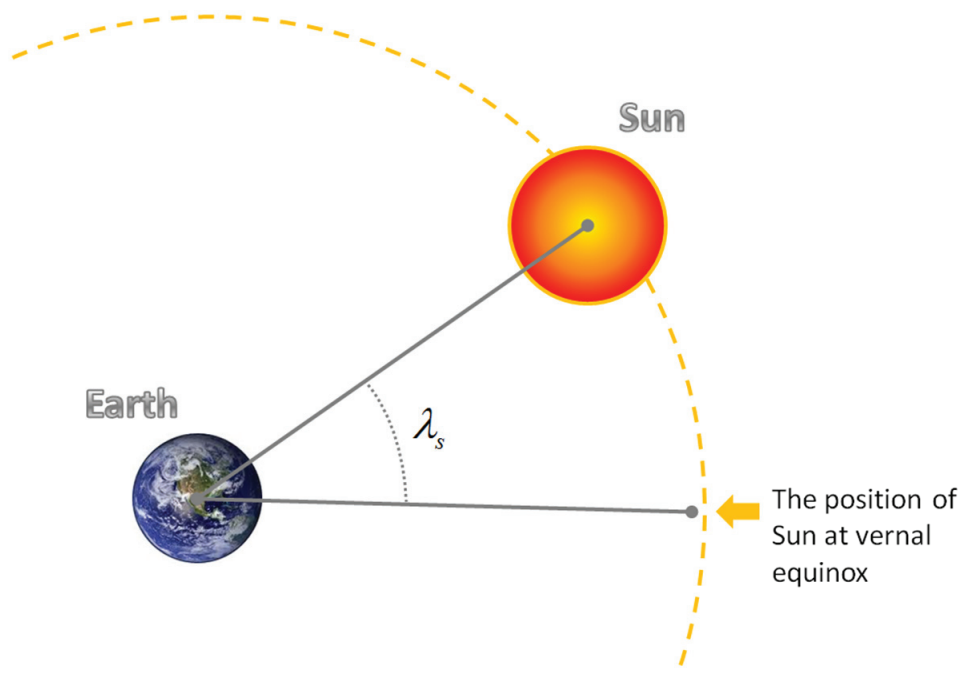

Figure 4. Sun position in imaginary orbit around Earth, , adapted from Reference 5. 
Both the Sun's elevation and position describe its imaginary rotation around the Earth. The position vector can be calculated as rotations:

$$
\left.\hat{S}\right|_{I}=\left[\begin{array}{c}
\cos \left(\varepsilon_{s}\right) \cos \left(\lambda_{s}\right) \\
\sin \left(\lambda_{s}\right) \\
\sin \left(\varepsilon_{s}\right) \cos \left(\lambda_{s}\right)
\end{array}\right]
$$

\section{Eclipse Model}

While orbiting the Earth, the spacecraft may pass into the Earth's shadow. The timing of the eclipse conditions depends on the orbital parameters. To determine when the satellite is in eclipse, we use the cylindrical shadow model. ${ }^{14,15}$ With this model, Earth's shadow is modeled as a cylinder of infinite length and radius equal to Earth's radius, $R_{E}$. Consider a unit shadow vector $\hat{u}_{s h}$ which is antiparallel to the inertial unit Sun vector, as shown in Eq. (16). This shadow vector lies on the center line of the shadow cylinder, as shown in Fig.5.

$$
\hat{u}_{s h}=-\left.\hat{S}\right|_{I}
$$

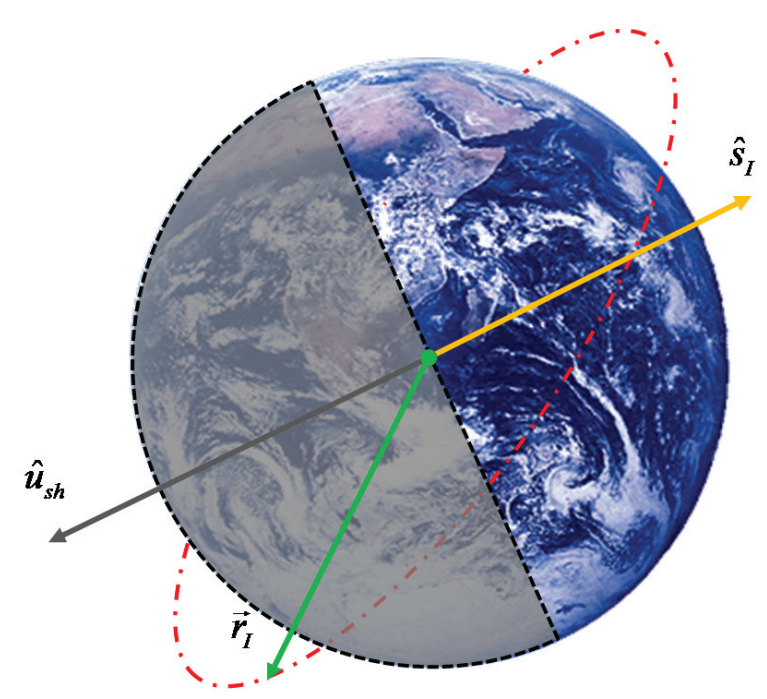

Figure 5. A unit eclipse vector in the Earth, adapted from Reference 14.

There are two conditions that determine if the spacecraft is in eclipse. First, the spacecraft must be located on the night side of the Earth. Mathematically, this is given by Eq. (17). Second, the spacecraft must be within the shadow cylinder, which is the case if Eq. (18) is true. In Eq. 18, selecting $R_{E}$ to be the equatorial rather than polar radius will generally results in slightly longer eclipse times than in reality ${ }^{14} .15$

$$
\begin{gathered}
\hat{u}_{s h} \cdot \hat{r}_{I}>0 \\
|R \cdot| \hat{u}_{s h} \times \hat{r}_{E C I}|| \mid \leq R_{E}
\end{gathered}
$$

\section{Attitude Control}

Next, we describe the attitude control block. Initially, we have implemented the model for a passive magnetic control system, but this block is modular so it can be replaced with other control schemes. The passive magnetic control system consists of permanent magnets, to align the satellite with Earth's magnetic field, and soft magnetic material (hysteresis strips), which dissipate energy via heat to dampen satellite rotation.

\section{A. Passive Stabilization System Modeling}

Passive attitude control is very efficient for small satellites from cost, weight, power, and reliability perspectives. For a small satellite which is passively magnetically controlled with a permanent magnet rod and hysteresis materials, the 
two components of the passive stabilization system serve different purposes, both of which are needed for passive stabilization. The model presented in this section has been used for the Radio Aurora Explorer (RAX) CubeSat mission. ${ }^{8}$

\section{Permanent Magnet}

The permanent magnet aligns the body z-axis with the Earth's magnetic field after deployment from the launch vehicle. As the Earth's geographic North Pole is its Magnetic South Pole, the north pole of the permanent magnet material will be pointed toward the Earth's North Pole. This will ensure that a satellite's antenna is Earth-pointing over the northern hemisphere, which is the desired attitude for the RAX mission.

A diagram of the pointing vector of the magnet and antenna is shown below in Fig. 6. Here, the arrow is the

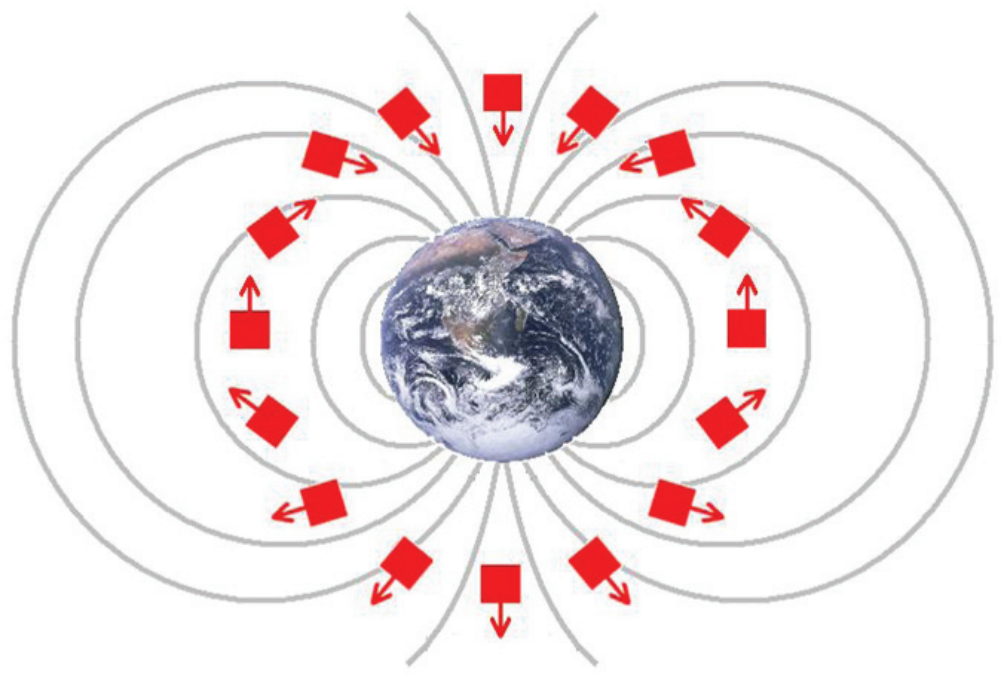

Figure 6. Passive magnetic stabilization description.

direction of the antenna, which is magnetic-field aligned due to the permanent magnet within the satellite. The torque generated by the permanent control magnet is given in Eq. (19), where $\vec{m}$ is the magnetic dipole moment vector and $\vec{m}$ is Earth's local magnetic field vector.

$$
\vec{M}=\vec{m} \times \vec{B}
$$

\section{Hysteresis Strips}

The hysteresis strips are made of soft magnetic material with high permeability and low coercivity. Materials with these characteristics induce a magnetic dipole of their own when they spin through a magnetic field. In many small satellite projects, these hysteresis strips are implemented in two directions, one along the x-axis and one along the $\mathrm{y}$-axis on body frame $F_{B}$ (assuming the permanent magnet is aligned with the z-axis). In this orientation, the hysteresis material will dissipate the satellite's rotational energy into heat while it spins about the body z-axis. Our development follows the model of hysteresis rods given in Park et al, ${ }^{7}$ which is based on the arc tangent graph. We provide a general dynamic description of each hysteresis rod in terms of a single ordinary differential equation that depends on several physical parameters that describe the magnetic properties of the hysteresis rod materials. The differential equation for each hysteresis rod clearly identifies the memory, namely the induced magnetic flux density in the hysteresis rod. The subsequent development assumes that the two hysteresis rods are physically well separated relative to each other and relative to the permanent magnet to avoid mutual magnetization. The component of the Earth's magnetic field in the direction of hysteresis $\operatorname{rod} i$ is

$$
H^{i}=\left.\hat{b}_{i}^{T} O_{B / E} \vec{H}\right|_{E}
$$

where $\hat{b}_{i}$ is the unit vector describing the direction of the $i$-th hysteresis strip in the body-fixed frame and $\left.\vec{H}\right|_{E}$ is the Earth magnetic field vector resolved in ECEF frame. The induced magnetic flux density in hysteresis rod $i$ is described 
by the differential equation :

$$
\begin{gathered}
\dot{B}^{i}=\frac{2 B_{m}}{H_{r} \pi}\left(\frac{H^{*} \cos \left(\frac{\pi B^{i}}{2 B_{m}}\right)-H_{r} \sin \left(\frac{\pi B^{i}}{2 B_{m}}\right)}{2 H_{c}}\right)^{2} \dot{H}^{i} \\
H^{*}=H^{i}+H_{c}\left(\dot{H}^{i} \geq 0\right) \\
H^{*}=H_{c}-H^{i}\left(\dot{H}^{i}<0\right)
\end{gathered}
$$

where,

$$
\dot{H}^{i}=\left.\hat{b}_{i}^{T}\left(-S(\omega) O_{B / I}\left(O_{E / I}\right)^{T}+O_{B / I}\left(\dot{O}_{E / I}\right)^{T}\right) \vec{H}\right|_{E}+\left.\hat{b}_{i}^{T} O_{B / I}\left(O_{E / I}\right)^{T} \dot{\vec{H}}\right|_{E}
$$

Here $B_{m}$ is the constant saturation value of the induced magnetic flux density of the hysteresis rod, $H_{c}$ is a constant coercivity value of the hysteresis material, and $H_{r}$ is a constant remanence value of the hysteresis material. ${ }^{7}$ Fig. 7 shows a typical plot of the time evolution of the hysteresis in the $B^{i}$ versus $H^{i}$ plane for $H^{i}(t)$. The magnetic torque

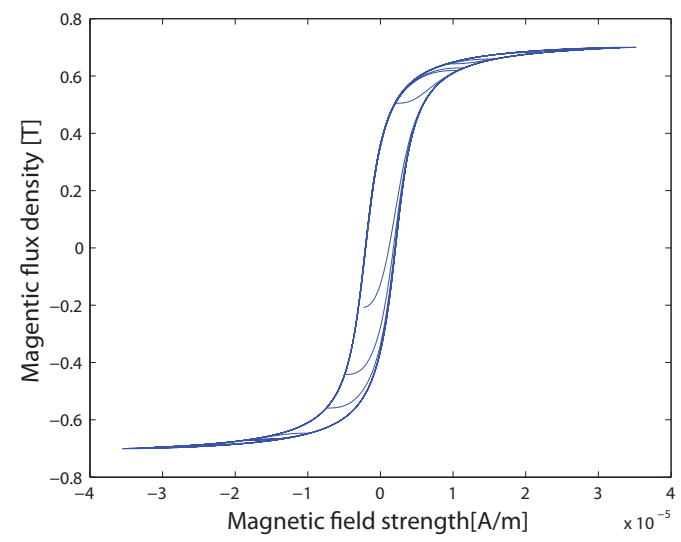

Figure 7. Typical Hysteresis Loop.

due to the presence of hysteresis rod $i$, expressed in the body-fixed frame, is

$$
\vec{M}_{i}=\vec{B}^{i} V_{h} \hat{b}_{i} \times\left. O_{B / E} \vec{H}\right|_{E}
$$

where $V_{h}$ is the volume of each hysteresis rod. The direction of the moment produced by hysteresis rod $i$ is orthogonal to the longitudinal axis of that hysteresis rod and to the direction of the Earth's magnetic field at the satellite's location.

\section{Disturbance Forces}

As motivated in the introduction, for high fidelity long period simulations, disturbance force effects must be considered. These disturbance forces can be categorized into orbital perturbation forces and rotational disturbance moments. Orbital perturbation forces modify the orbit trajectory, while rotational disturbance moments modify the attitude.

\section{A. Orbital Perturbation Forces}

The most important orbital perturbation forces of a satellite are earth oblateness effects, the aerodynamic drag, and the solar pressure. In this section, these forces are explained and their implementation to the simulator explained. With the growing trend toward reducing the size of satellites, careful modeling the perturbation forces is necessary. ${ }^{16}$

\section{Earth Oblateness Effects}

The Earth oblateness affects a satellite's acceleration. We model this effect by taking the gradient of the gravitational potential function as a function of position to calculate the gravity force in the orbit propagator. The total gravity 
potential function, which includes oblateness effects can be defined at any position as,

$$
\begin{gathered}
U_{\text {total }}=U_{\text {gravity }}+U_{\text {oblate }}=\frac{\mu}{r}\left[1-\sum_{n=2}^{\infty} J_{n} \cdot\left(\frac{R_{E}}{r}\right)^{n} \cdot P_{n} \cdot \sin (L)\right] \\
U_{\text {oblate }}=\frac{\mu}{r}\left[-\sum_{n=2}^{\infty} J_{n} \cdot\left(\frac{R_{E}}{r}\right)^{n} \cdot P_{n} \cdot \sin (L)\right]
\end{gathered}
$$

where $U_{\text {gravity }}$ is Newton's 2-body gravity term, $\mu=G M$ is Earth's gravitational constant, $R_{E}$ is Earth's equatorial radius, $P_{n}$ are Legendre polynomials, $L$ is geocentric latitude, and $J_{n}$ are dimensionless geopotential coefficients and the first three term which is most dominant are:

$$
J_{2}=0.00108263, J_{3}=-0.00000254, J_{4}=-0.00000161
$$

The gradient of the gravitational potential function Eq. (25) can be divided into a gravitational force and gravitational disturbance terms. We define gravitational disturbance as $\vec{F}_{\text {oblate }}$,

$$
\vec{F}_{\text {oblate }}=\frac{\partial U_{\text {oblate }}}{\partial r} \cdot \frac{\vec{r}}{r}
$$

where $\vec{r}$ is the satellite position vector and $r$ is the magnitude of the vector $\vec{r}$.

\section{Solar Pressure}

A satellite in low earth orbit (LEO) is affected by three major electromagnetic radiation sources in space. These sources are the sun, solar radiation reflected by the Earth (termed albedo), and the thermal infrared radiation of the Earth. In this paper, only the first source, direct sun radiation, is modeled as a disturbance forces as it is the largest in magnitude. The sun's electromagnetic radiation exerts a normal force on space objects, known as solar radiation pressure. The concept of solar radiation force is modeled,

$$
\begin{gathered}
\vec{F}_{\text {Solar }}=\sum_{k=1}^{n} \vec{F}_{\text {Solar }, k} \\
\vec{F}_{\text {Solar }, k}=-P_{S} \cdot A_{k} \cdot \cos \left(\theta_{k}\right)\left[\left.(1-\varepsilon) \hat{S}\right|_{B}+\left.2 \varepsilon \cos \left(\theta_{k}\right) \hat{n}_{k}\right|_{B}\right] \\
\cos \left(\theta_{k}\right)=\left.\left.\hat{S}\right|_{B} \cdot \hat{n}_{k}\right|_{B}
\end{gathered}
$$

In Eq. (28) - (30), $n$ is the number of satellite surfaces which has the effective angle $\left(0-90^{\circ}\right)$ with the sun vector, $P_{S}$ is solar radiation pressure near Earth and its value is $4.56 \times 10^{-6} \mathrm{~N} / \mathrm{m}^{2}, A$ is the exposed area of the satellite to Sun, $\left.\hat{S}\right|_{B}$ is a unit sun direction vector on body frame, $\left.\hat{n}_{k}\right|_{B}$ is the normal vector of exposed $k^{\text {th }}$ face on body frame, $\varepsilon$ is reflectivity and $A_{k}$ is the area of the $k^{\text {th }}$ surface. ${ }^{17}$

\section{Aerodynamic Drag}

Aerodynamic drag is the restraining force that acts on the satellite surfaces in the direction of the air flow and it can be calculated,

$$
\begin{gathered}
\vec{F}_{\text {Aero }}=\sum_{k=1}^{n} \vec{F}_{\text {Aero }, k} \\
\vec{F}_{\text {Aero }, k}=-\frac{1}{2} C_{D} \cdot A_{D, k} \cdot \rho \cdot v_{r}{ }^{2} \frac{\vec{v}_{r}}{v_{r}}
\end{gathered}
$$

Here, we define $\vec{F}_{\text {Aero }, k}$ is aerodynamic drags of each surface of the satellite, $\vec{v}_{r}=\vec{v}_{E C I}-\vec{\omega}_{E} \times \vec{r}_{E C I}$ and can also assume $\vec{\omega}_{E} \approx 15^{\circ} / \mathrm{hr}$ based on the simple calculation that the Earth's rotation period is 24 hours. $C_{D}$ is the drag coefficient and it depends on the geometry and size of faces of the satellite, $\rho$ is the air density and depends on the altitude and $A_{D, k}$ is the $k^{\text {th }}$ area exposed to ram directions.

These forces we have introduced can be added and construct total disturbance forces as in Eq. (33)

$$
\vec{F}_{\text {Disturbance }}=\vec{F}_{\text {oblate }}+\vec{F}_{\text {Aero }}+\vec{F}_{\text {Solar }}
$$

By adding these orbital perturbations to the standard Earth gravitational force from the 2-body problem, it is possible to calculate the position and velocity of the satellite with an improved accuracy. 


\section{B. Disturbance Moments}

In this section, we discuss disturbance moments which effect the attitude of the satellite. Disturbance moments include the moments generated by solar pressure, aerodynamic drag effect, gravity gradient effect and residual dipole. The solar pressure and the aerodynamic drag generate moments when there is a non-zero distance between the geometric center and the center of mass, which is true in most practical systems.

\section{Gravity Gradient Disturbance}

If the mass of the satellite is distributed unevenly about the geometric center, the force from the Earth's gravity will pull unevenly on the satellite. This gives rise to the gravity gradient moment,

$$
\vec{\tau}_{G . G}=3 \frac{G M}{R^{3}} \vec{u}_{\text {nadir }} \times J \cdot \vec{u}_{\text {nadir }},
$$

where $J$ is a inertia matrix and $\vec{u}_{\text {nadir }}=\hat{k}_{O}$. This $\vec{u}_{\text {nadir }}$ is resolved in the body-fixed frame as,

$$
\begin{aligned}
\left.\vec{u}_{\text {nadir }}\right|_{B}=\left.\hat{k}_{L}\right|_{B} & =\left.O_{B / L} \cdot \hat{k}_{L}\right|_{L}, \\
& =O_{B / L} \cdot\left[\begin{array}{lll}
0 & 0 & 1
\end{array}\right]^{T},
\end{aligned}
$$

where the subscript $B$ indicates the body-fixed frame and the subscript $L$ indicates the LVLH frame.

\section{Solar Pressure Disturbance}

If there is a non-zero distance between the geometric center and the center of mass, this pressure causes a solar pressure disturbance moment. The following method is used to calculate this moment. ${ }^{14}$

1. Calculate $\left.\hat{S}\right|_{B}$, the unit vector in the Sun direction resolved on the body-fixed frame and $\left.\hat{n}_{k}\right|_{B}$, $k$ th the normal vectors of spacecraft surfaces.

2. Calculate the angle $\theta_{k}$ between $\left.\hat{S}\right|_{B}$ and $\left.\hat{n}_{k}\right|_{B}$ using dot products.

3. Eliminate the effect of the surfaces which are not illuminated by the Sun. (which could be checked with $\theta_{k}$ of the previous step and this lager than 90 degree angle means that this surface is not exposed to Sun).

4. Calculate $A_{S, k}$, the projected area of each surface to the Sun direction and calculate $\vec{F}_{\text {Solar }, k}$, the pressure force vector of each surface.

5. Calculate $\vec{r}_{G C, k / C o M}$, vectors from the center of mass of satellite to the geometric center of the illuminated surface.

6. Calculate $\vec{\tau}_{\text {Solar }}$, the moment of each surface with cross product with the pressure force of step 3 and the distance vector of step 4 .

Based on this procedure, we obtain Eq. (36) - (36), ${ }^{14}$

$$
\begin{gathered}
\vec{\tau}_{\text {Solar }}=\sum_{k=1}^{n}\left(\vec{r}_{G C, k / C o M} \times \vec{F}_{\text {Solar }, k}\right) \\
\vec{F}_{\text {Solar }, k}=-P_{S} \cdot A_{S, k} \cdot \cos \left(\theta_{k}\right)\left[\left.(1-\varepsilon) \hat{s}\right|_{B}+\left.2 \varepsilon \cos \left(\theta_{k}\right) \hat{n}_{k}\right|_{B}\right]
\end{gathered}
$$

where, $\vec{r}_{G C, k / C o M}$ is the vector from the center of mass to the area center of the $k^{\text {th }}$ surface. $n$ is the number of the surface which has the effective angle $\left(0-90^{\circ}\right)$ with the sun vector. 


\section{Aerodynamic Drag}

Atmospheric drag is a source of disturbance moments on a spacecraft in the low part of LEO due to atmospheric density of that region. If there is a non-zero distance between the geometric center and the center of mass, this pressure causes a disturbance moment which can be modeled using the same approach as the one for the solar pressure.

$$
\begin{gathered}
\vec{\tau}_{\text {Aero }}=\sum_{k=1}^{n}\left(\vec{r}_{G C, k / C o M} \times \vec{F}_{\text {Aero }, k}\right) \\
\vec{F}_{\text {Aero }, k}=-\frac{1}{2} C_{D} \cdot A_{D, k} \cdot \rho \cdot v_{r} \frac{\vec{v}_{r}}{v_{r}}
\end{gathered}
$$

For $\rho$ value, we used the Harris Priester model which calculates $\rho$ based on the altitude.

\section{Residual Dipole}

In LEO, the interaction between the Earth's magnetic field and the magnetic dipole of a satellite generates a moment. Many satellites take advantage of this by using either electromagnets or permanent magnets for spacecraft attitude control. In addition to the designed magnetic dipole, all spacecraft have an unintended dipole, known as a residual dipole, that is caused by electric currents and magnetic material within the satellite. The moment caused by the interaction between the residual dipole and Earth's magnetic field is known as the residual dipole moment, and is described by Eq. (40).

$$
\vec{T}_{r}=\vec{m}_{r} \times \vec{B}
$$

In Eq. (40), $\vec{m}_{r}$ is the magnetic moment of the residual dipole, and $\vec{B}$ is Earth's local magnetic field. For small satellites, the residual dipole moment can be the dominant disturbance moment. ${ }^{18}$

\section{Simulation Results}

To show applicability of the simulator, we show simulation results with the representative example, the Radio Aurora eXplorer (RAX) small satellite from the University of Michigan. ${ }^{8}$ The satellite orbit is circular with an altitude of $650 \mathrm{~km}$ and an inclination of $72^{\circ}$. To demonstrate high fidelity simulation results, we show the effect of orbital perturbations, compare the RK45 and LGVI integration schemes, and show the effects of disturbance moments.

\section{A. Orbital Perturbation Effects}

When we only consider the Earth's oblateness effects in our orbit simulation, the orbital radius is as shown in Fig. 8 and compared to the simulation result which considers all other orbital perturbations such as aerodynamic drag and solar pressure to asses the impact on orbit propagation. In Fig. 9, the blue graph is the one which only includes the Earth oblateness effect and the red graph is the simulation result of all disturbance forces are included.

To asses the disturbance force effect clearly, a long simulation period was needed, therefore we show a simulation period of 2,000 orbits. In the case of RAX, the orbital period is about 98 minutes (5863.7 second) therefore the total simulation time is about 136 days.

To asses the effect of the aerodynamic drag and solar pressure disturbances on the orbit, we now include these disturbances in our simulation, and the orbit radius graph comparing the orbit with and without these disturbances is shown in Fig. 9. When disturbance forces are small, such as in the first 10 orbits, the disturbance effects on the orbit radius is not visible in Fig 8. However, as simulation time increases, the results with all disturbance forces results in a difference in orbit radius relative to the simulation result which only includes the Earth oblateness effect, as shown in Fig. 9. Therefore, we need to consider these disturbance effect on the orbit to accurately estimate the satellite position and the orbit lifetime of a satellite.

\section{B. Comparison Between Runge-Kutta and Lie Group Variational Integrator}

In the passive stabilization system, only a small rate of the kinetic energy is dissipated by hysteresis. However, if the fidelity of simulator is low, it is not easy to evaluate the performance of the passive stabilization system because the simulation error can be combined with hysteresis effects of the system. To prevent this, our simulator adopts the LGVI and to prove the effectiveness of the simulator, we compare the two different integration schemes, Runge-Kutta45 (RK45) and LGVI, with a same configuration. We focused on the kinetic energy level and the stabilization time of the passive magnetic stabilization system to prove the high fidelity of LGVI. 


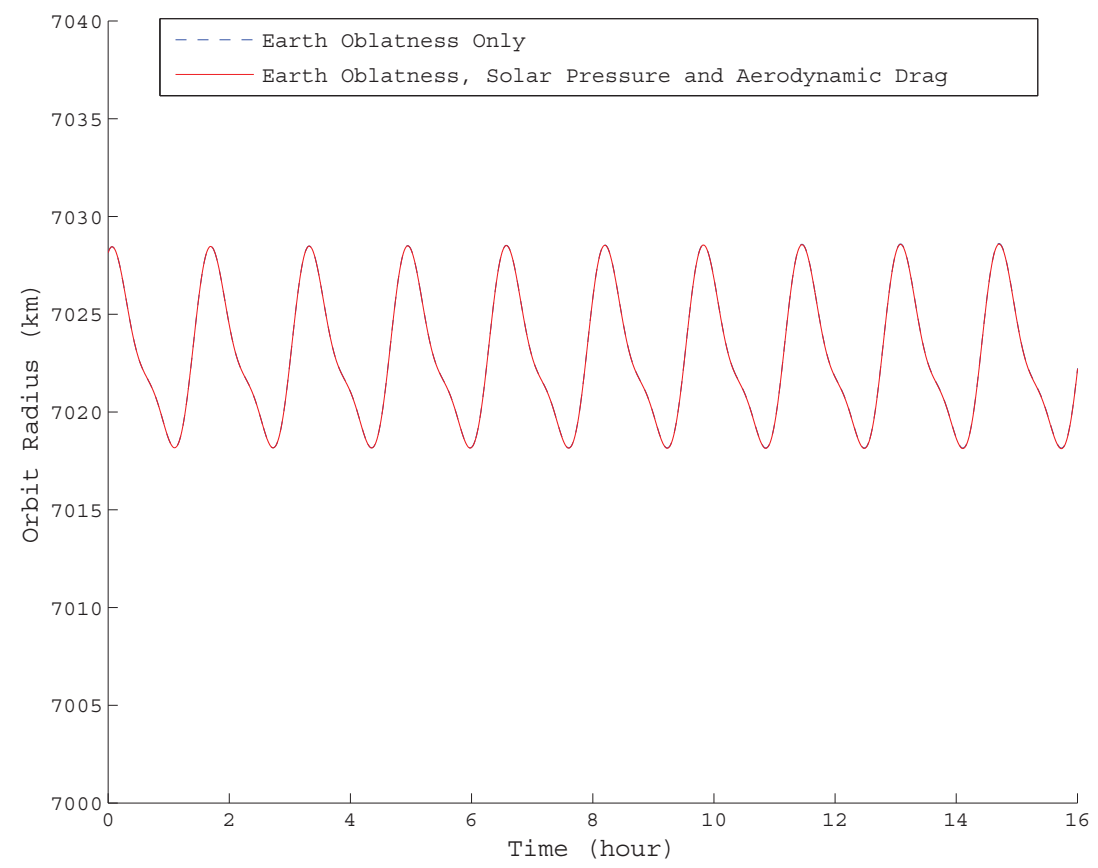

Figure 8. RAX orbit radius simulation (initial 10 orbits). The difference between two case is negligible.

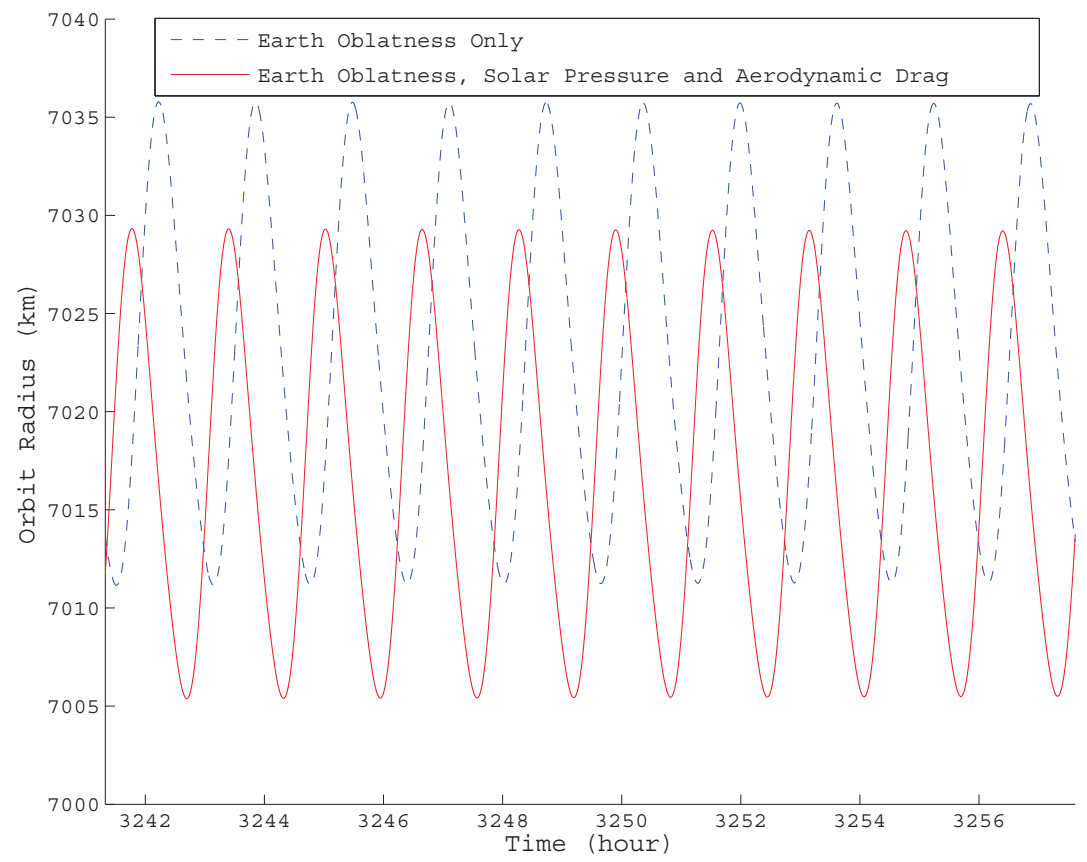

Figure 9. RAX orbit radius simulation (after 2000 orbits $\approx 136$ days). Orbit perturbation forces results in large orbit radius differences. 


\section{Without hysteresis effects}

We first compare the difference between the quaternion integration based RK45 method and LGVI in the case of no hysteresis effects. We ran the simulation for 400 orbits to compare the energy preservation property of LGVI and RK45 integration in Fig. 10 and Fig. 11. The quaternion integration based RK45 results in a loss of kinetic energy because this method is not sophisticated enough to preserve the angular momentum of the rotational motion. In the case of RK45, the kinetic energy loss is dependent on the integration time interval width. If the time interval width is small, the kinetic energy loss ratio of RK45 is also small and shows no difference relative to the higher fidelity LGVI. However, by increasing time interval, the energy loss ratio grows significantly using the RK45 integration scheme relative to the LGVI integration scheme. One other merit of the LGVI is integration speed. RK45 can achieve the same kinetic energy loss as LGVI approach by choosing a small integration time interval. However, with the same time interval size, LGVI's integration speed is about 3.8 times faster than that of RK45. Based on this characteristic, the developed simulator was able to handle long period simulations with a relatively larger time step of $0.2 \mathrm{sec}$ and $0.5 \mathrm{sec}$. The simulation for 400 orbits with no hysteresis effect is plotted in Fig. 10 and Fig. 11. If we use a small time interval such as $0.1 \mathrm{sec}$, the simulation result difference between LGVI and RK45 will be almost zero, but the integration calculation time is much longer with the RK45 integrator relative to the LGVI. Table 1 shows the simulation time comparison between LGVI and RK45.

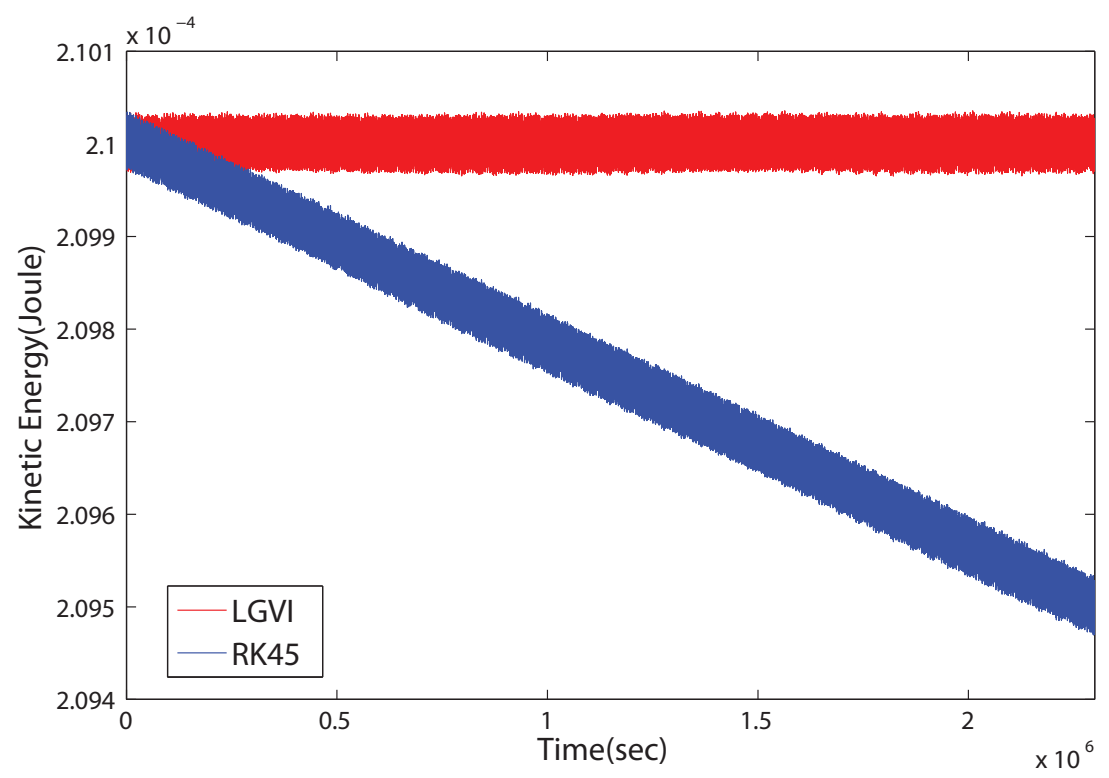

Figure 10. The Simulation with no hysteresis effect ( $T_{s}=1.0 \mathrm{sec}, 400$ orbits).

\begin{tabular}{|c|c|c|c|c|}
\hline & \multicolumn{2}{|c|}{10 orbits } & \multicolumn{2}{c|}{100 orbits } \\
\hline sample time interval & LGVI & RK45 & LGVI & RK45 \\
\hline $0.5 \mathrm{sec}$ & $157.20 \mathrm{sec}$ & $609.78 \mathrm{sec}$ & $1598.28 \mathrm{sec}$ & $6176.88 \mathrm{sec}$ \\
\hline $0.2 \mathrm{sec}$ & $386.57 \mathrm{sec}$ & $1487.52 \mathrm{sec}$ & $3930.34 \mathrm{sec}$ & $15068.12 \mathrm{sec}$ \\
\hline
\end{tabular}

Table 1. The integration time comparison between LGVI and RK45.

\section{Considering Hysteresis Effects}

Next we examine the energy dissipation effect when hysteresis in present to estimate the stabilization time of the passive magnetic attitude control system.

To compare LGVI and RK45 based integration with the hysteresis effect, we have run simulations for 500 orbits to find the duration of time such that most of kinetic energy is dissipated. The simulation result is presented in Fig. 12 - 13, where the RK45 integration kinetic energy loss error is combined with the energy dissipation effect of the 


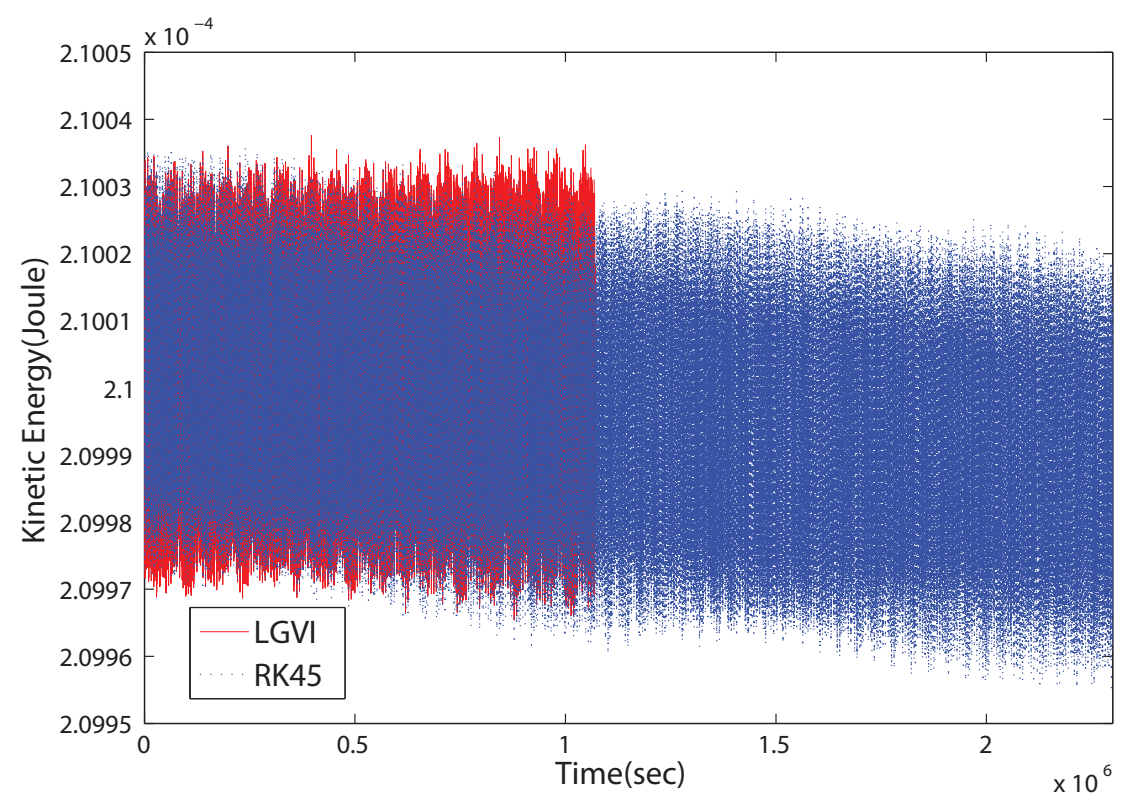

Figure 11. The Simulation with no hysteresis effect $\left(T_{s}=0.5 \mathrm{sec}, 400\right.$ orbits).

hysteresis model. As explained, RK45 do not preserve the kinetic energy, the stabilization time estimation with the RK45 is different with the LGVI.

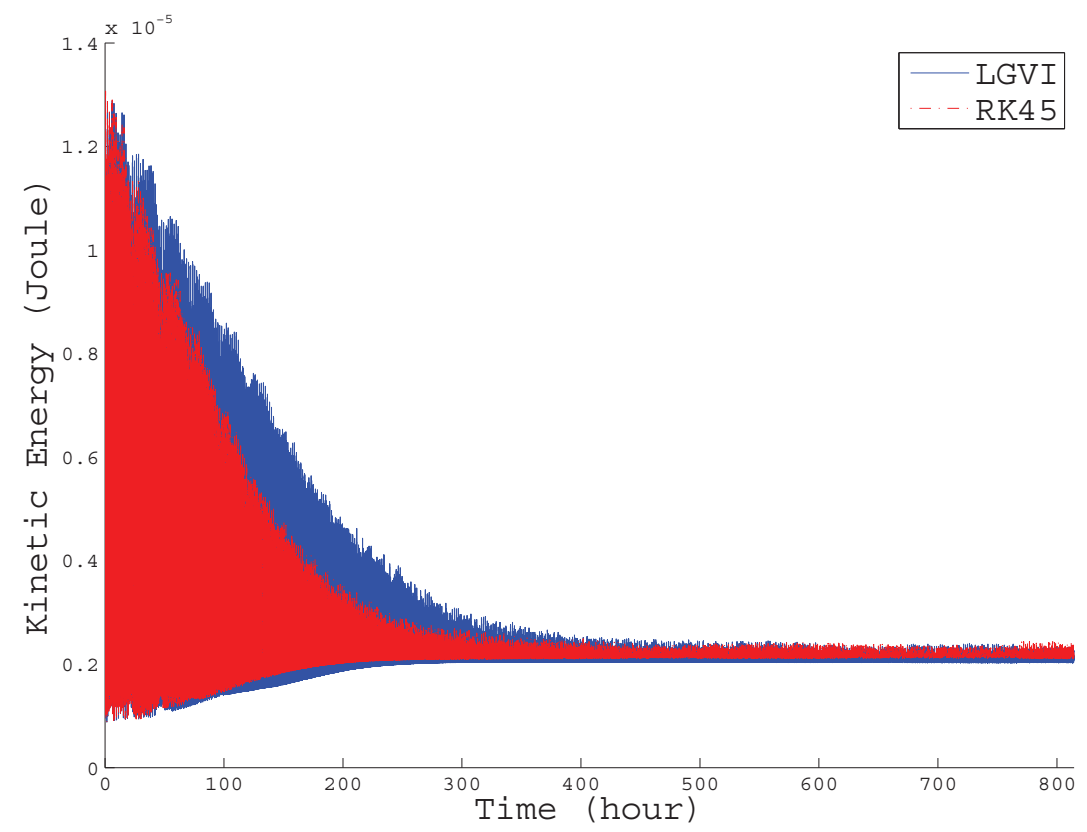

Figure 12. Difference of two integration schemes simulation results with hysteresis $\left(T_{s}=1.0 \mathrm{sec}\right)$.

\section{Disturbance Moment Simulation Result}

In this section, the disturbance moment simulation result is presented. The expected magnitude range of each disturbance force is given by the simulation results. The simulation depends on various parameters, and the relevant 


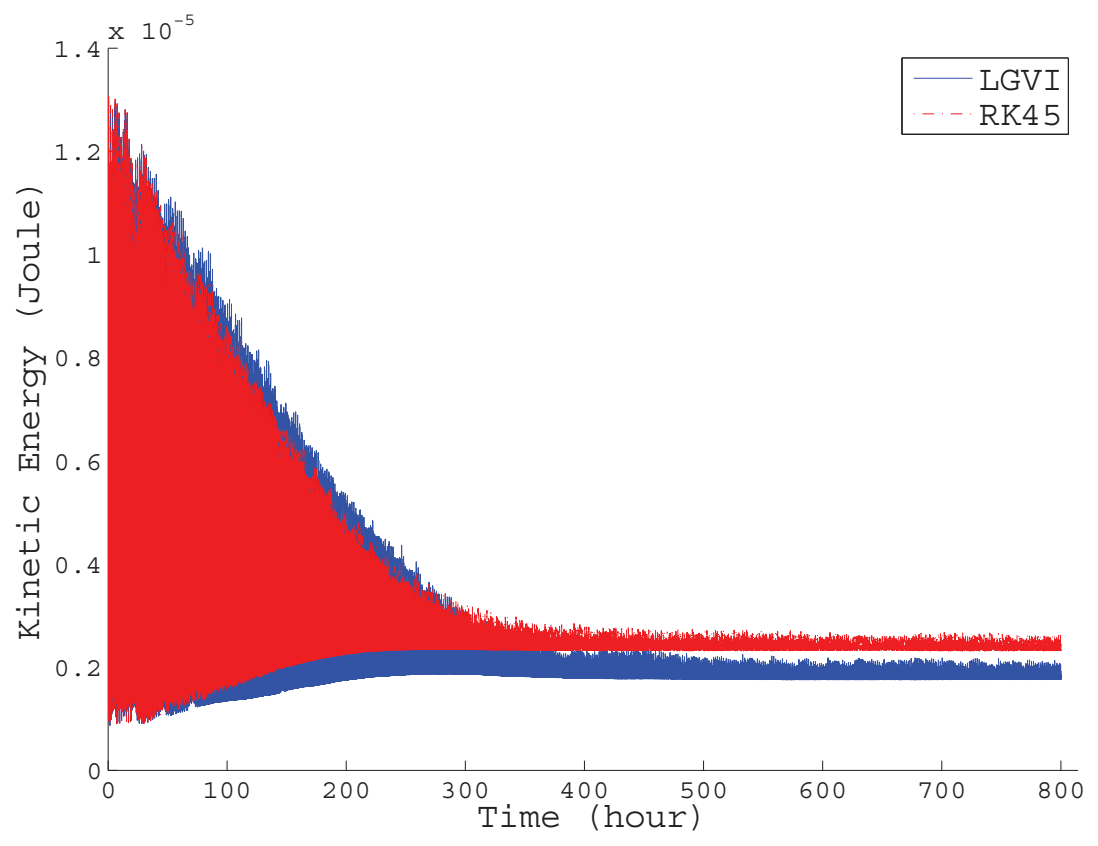

Figure 13. Difference of two integration schemes simulation results with hysteresis $\left(T_{s}=0.5 \mathrm{sec}\right)$ : The difference between the two integration schemes is smaller than the difference with a larger simulation time interval in Fig. 12.

parameters are listed below. The mass properties are the pre-flight measurements of the RAX spacecraft.

The aerodynamic drag coefficient comes from the estimated CubeSat drag coefficient of Reference.19. solar radiation Ref.20, reflectivity effect Ref.21. The RAX residual dipole has not been characterized, so we use the residual dipole estimate of the PACE spacecraft, ${ }^{22}$ which is a $1 \mathrm{U}$ CubeSat.

- The mass: $m=2.83616 \mathrm{~kg}$

- The moment of inertia:

$$
J=\left[\begin{array}{ccc}
0.018 & 0 & 0 \\
0 & 0.018 & 0 \\
0 & 0 & 0.006
\end{array}\right]\left(\mathrm{kg} \cdot \mathrm{m}^{2}\right)
$$

- The distance between the geographical center and the center of mass:

$$
\left.\vec{r}_{C o M / G C}\right|_{B}=\left[\begin{array}{lll}
0.001 & 0 & -0.009
\end{array}\right]^{T}(m)
$$

- The aerodynamic drag coefficient: $C_{D}=2.0$

- The solar radiation pressure near Earth (at $1 \mathrm{AU}$ ): $P_{S}=4.56 \times 10^{-6} \mathrm{~N} / \mathrm{m}^{2}$

- The reflectivity effect factor: $\varepsilon=0.21$

- The residual dipole vector:

$$
\vec{M}_{\text {residual }}=\left[\begin{array}{lll}
0 & 0.0005 & 0
\end{array}\right]^{T}\left(A \cdot m^{2}\right)
$$

\section{Norms of Each Disturbance Moments}

To evaluate the total effect of each disturbance moment, Euclidean norms moments are calculated. With RAX simulation configuration, Fig. 14 shows that residual dipole and the gravity gradient moments are most dominant. The moments generated by aerodynamic drag are almost negligible at the altitude of $650 \mathrm{~km}$. 


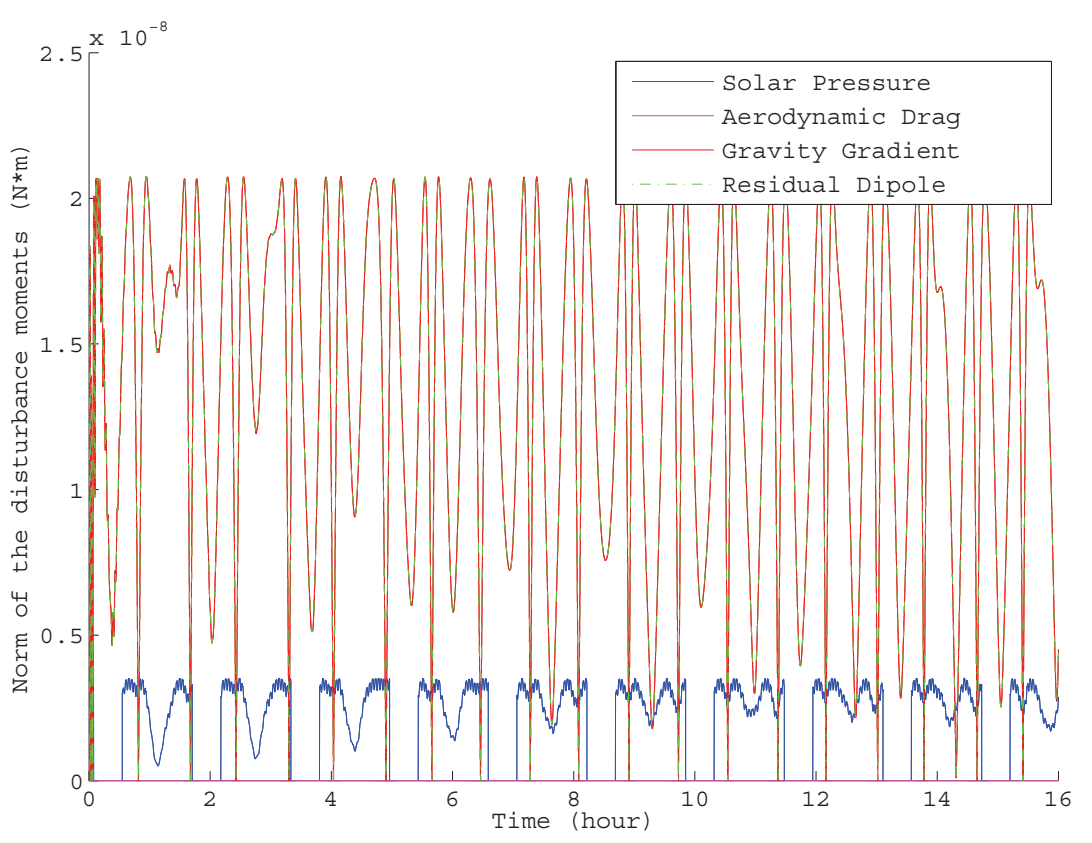

Figure 14. Norms of each disturbance moments (10 orbits): The dominant disturbance moments are generated by the residual dipole and the gravity gradient.

\section{Gravity Gradient Disturbance Moment}

Fig. 15 shows the simulation result of the gravity gradient disturbance moment. The gravity gradient moment is only dependent on the moment of inertia.. Many satellites have a boom or antenna which affect to the gravity gradient disturbance moment. An accurate value of the moment of inertia make it possible to asses the disturbance effect of a boom or antenna. By checking the range of this moment, we expect that the gravity gradient moment will be one of the dominant moments in the system as RAX is $3 \mathrm{U}$ small satellite which $\mathrm{z}$-axis length is three times longer than $\mathrm{X}$-axis or y-axis length.

\section{Solar Pressure Disturbance Moment}

Fig. 16 shows the simulation result of the solar pressure disturbance moment. As explained previous section, the solar pressure disturbance moment is largely dependent on the distance between the geographical center and the center of mass. Depending on the assumption about this distance, the disturbance moment varies. Furthermore, solar pressure is also a function of eclipse, as seen in Fig. 16.

\section{Aerodynamic Drag Disturbance Moment}

Fig. 17 shows the simulation result for the aerodynamic drag moment. Similar to the solar pressure disturbance moment, the aerodynamic drag moment is dependent on the distance between the geographical center and the center of mass. Aerodynamic drag is also a function of the density, which is dependent on the altitude. This simulator makes it possible to evaluate the influence of different altitude profiles and expected atmospheric densities on aerodynamic drag disturbance moment. By evaluating the minimum and maximum value of this moment, we note that for the RAX configuration that the aerodynamic drag moment is almost negligible relative to the others.

\section{Residual Dipole Disturbance Moment}

Fig. 18 shows the simulation result of the residual dipole moment. This is the most difficult disturbance moment to evaluate accurately because this is largely influenced by the configuration of the spacecraft hardware and also by time varying properties such as the electric power usage. The graph illustrates that the moment generated by the residual dipole only effects the $\mathrm{x}$ and $\mathrm{y}$ axis after the initial release. 


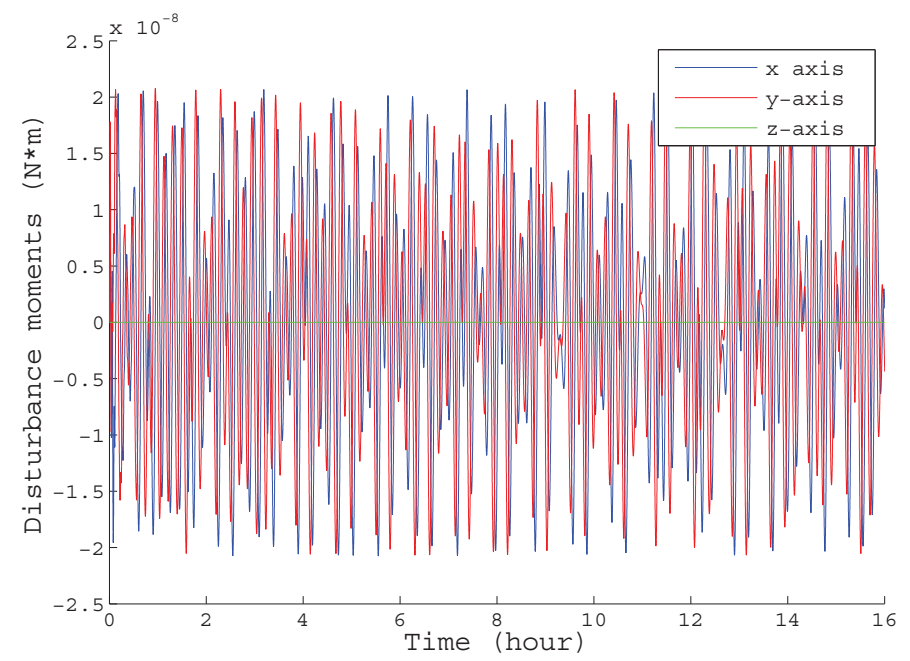

Figure 15. The gravity gradient disturbance moment (10 orbits).

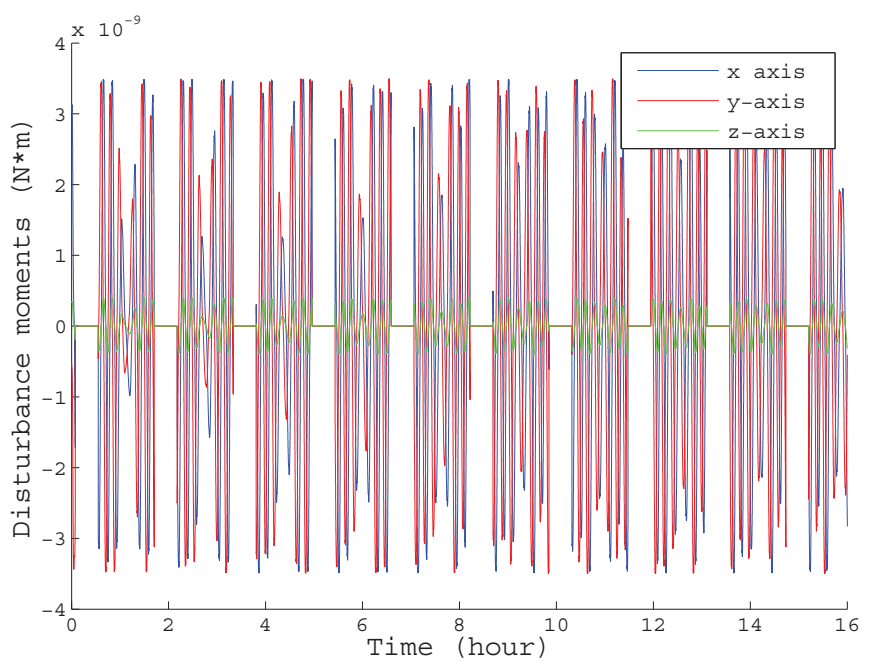

Figure 16. The solar pressure disturbance moment (10 orbits). 


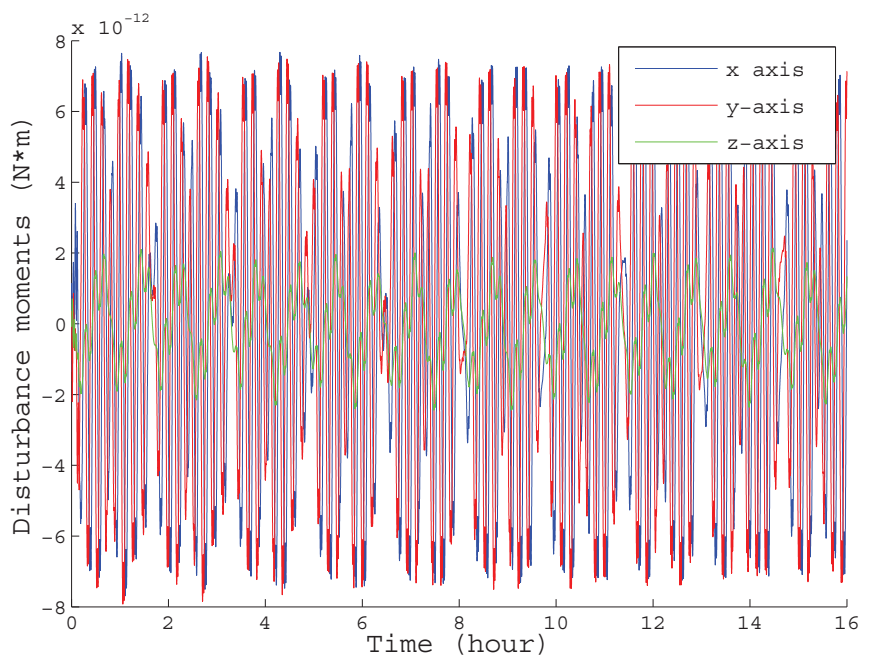

Figure 17. The aerodynamic drag disturbance moment (10 orbits).

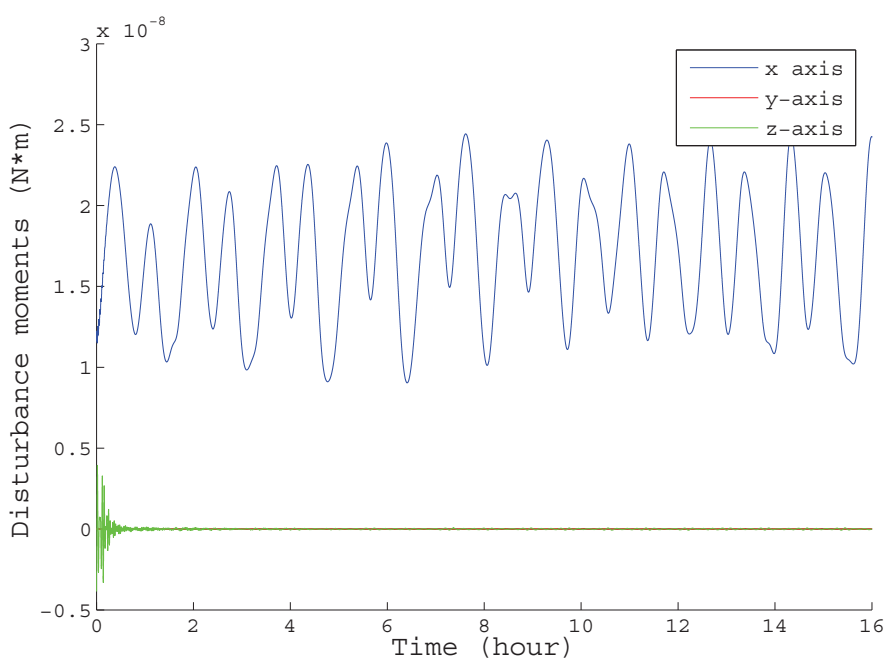

Figure 18. The residual dipole disturbance (10 orbits).

19 of 20

American Institute of Aeronautics and Astronautics 


\section{Conclusion}

We have developed a new satellite simulator with high fidelity nominal and perturbation dynamics which uses a Lie Group Variational Integrator. This simulation approach provides more accurate integration results and information relative to conventional simulators. In the design of the passive magnetic attitude stabilization system and other systems which use hysteresis, we can model the effect of hysteresis more accurately with our simulator. In other simulators, the quaternion integration based on RK45 is often used. This integration method does not preserve energy therefore, errors may accumulate and interfere with the true hysteresis effects. This high fidelity simulator enables the accurate estimation of stabilization time for passive magnetic attitude stabilization systems due to our integration scheme and consideration of disturbance forces and moments. With this simulator, a unified modeling approach to the satellite design process and dynamic modeling is possible and customizable to enable subsystem optimization. It will also help the analysis of further acquired data from small satellites, such as the RAX CubeSat. Future research topics with this simulator include analyzing the disturbance effects on the orbit for small satellites, as the orbits and attitude of small satellites can be greatly affected by these small disturbance forces.

\section{References}

${ }^{1}$ Pulecchi, T., Casella, F., and Lovera, M., "Object-oriented modelling for spacecraft dynamics: Tools and applications," Simulation Modelling Practice and Theory, Vol. 18, No. 1, 2010/01/, pp. 63 - 86.

${ }^{2}$ Turner, A. J. and Hall, C. D., "An Open-Source, Extensible Spacecraft Simulation and Modeling Environment Framework," AAS/AIAA Astrodynamics Specialists Conference, AAS/AIAA, August 3-7 2003, pp. AAS 03-501.

${ }^{3}$ Krogh, K., Attitude Determination for AAU CubeSat, Master's thesis, Department of Control Engineering, Aalborg University Institude of Electronic Systems, Denmark, June 2002.

${ }^{4}$ Rawashdeh, S. A. and Lumpp Jr., J. E., "Nano-satellite passive attitude stabilization systems design by orbital environment modeling and simulation," AIAA Infotech at Aerospace 2010, 2010.

${ }^{5}$ Svartveit, K., Attitude determination of the NCUBE satellite, Master's thesis, Department of Engineering Cybernetics, Norwegian University of Science and Technology, 2003.

${ }^{6}$ Lee, T., McClamroch, N. H., and Leok, M., "A lie group variational integrator for the attitude dynamics of a rigid body with applications to the 3D pendulum," 2005 IEEE International Conference on Control Applications, CCA, Toronto, ON, Canada, 2005, pp. $962-967$.

${ }^{7}$ Park, G., Seagraves, S., and McClamroch, N. H., "A Dynamic Model of a Passive Magnetic Attitude Control System for the RAX Nanosatellite," Proceedings of the AIAA Guidance, Navigation, and Control Conference, Toronto, Canada, 2010.

${ }^{8}$ Cutler, J., Bennett, M., Klesh, A., Bahcivan, H., and Doe, R., "The Radio Aurora Explorer A Bistatic Radar Mission to Measure Space Weather Phenomenon," Proceedings of the Small Satellite Conference, 2010.

${ }^{9}$ Lee, T., McClamroch, N. H., and Leok, M., "Lie group variational integrators for the full body problem," Computer Methods in Applied Mechanics and Engineering, Vol. 196, No. 29-30, 2007, pp. 2907-2924.

${ }^{10}$ Iserles, A., Munthe-Kaas, H. Z., Norsett, S. P., and Zanna, A., "Lie-group methods,” Vol. 9, 2000, pp. 215-365.

${ }^{11}$ Marsden, J. E. and West, M., "Discrete mechanics and variational integrators," Vol. 10, 2001, pp. 357-514.

${ }^{12}$ Macmillan, S. and Maus, S., "International Geomagnetic Reference Field - the tenth generation,” Vol. 57, No. 12, 2005, pp. $1135-40$.

${ }^{13}$ Svartveitk, K., Attitude determination of the NCUBE satellite, Master's thesis, Norwegian University of Science and Technology, Trondheim, Norway, Jan. 2003.

${ }^{14}$ Gieelmann, J., Development of an Active Magnetic Attitude Determination and Control System for Picosatellites on highly inclined circular Low Earth Orbits, Master's thesis, The Royal Melbourne Institute of Technology(RMIT), Melbourne, Australia, 2006.

${ }^{15}$ Vallado, D., Fundamentals of Astrodynamics and Applications, McGraw-Hill, New York, 2001.

${ }^{16}$ Atchison, J. A. and Peck, M. A., "A passive, sun-pointing, millimeter-scale solar sail,” Acta Astronautica, Vol. 67, No. 1-2, 2010 , pp. 108 121.

${ }^{17}$ Soop., E. M., Handbook of Geostationary orbits, Space Technology Library, 1994.

${ }^{18}$ Springmann, J. C., Cutler, J. W., and Bahcivan, H., "Magnetic Sensor Calibration and Residual Dipole Characterization for Application to Nanosatellites," AIAA/AAS Astrodynamics Specialist Conference Proceedings, Toronto, Canada, 2010.

${ }^{19}$ Maessen, D., van Breukelen, E., van Breukelen, E., and Bergsma, O., "Development of a Generic Inflatable De-Orbit Device for CubeSats," Proceedings of the International Astronautical Congress, Vol. 3, September 2007, pp. 1860-1870.

${ }^{20}$ Wie, B., Space Vehicle Dynamics and Control, AIAA, 1801 Alexander Bell Drive, Reston, VA 20191, 2008.

${ }^{21}$ Delgad, M. R., "Radiation Pressure Modeling the Space Environment," Tech. rep., Universidad Politecnica de Madrid, 2008.

${ }^{22}$ Scholz, A., Implementation of Advanced Attitude Determination and Control Techniques into a Nanosatellite, Master's thesis, Tainan, Taiwan, 1997. 